\title{
WIRELESS MICROPHONES INTERFERENCE DECREASING USING LTE FILTERS
}

\author{
Jovan Zlatanović, *, \\ Marina Marjanović, \\ Zoran Trivić
}

\author{
${ }^{1}$ PE Transmitters and Communications, \\ Belgrade, Serbia \\ ${ }^{2}$ Singidunum University, \\ Belgrade, Serbia
}

Correspondence:

Jovan Zlatanović

e-mail:

jovan.zlatanovic@etv.rs

\begin{abstract}
:
Strong signals from mobile phones can disturb and interfere with television signals making the channel impossible to receive the signal. In this paper, we measured the interference between wireless microphones and mobile phones that work in the same frequency Bandwidth from $750 \mathrm{MHz}$ to $850 \mathrm{MHz}$. The measurements were performed on Košutnjak site from Radio Television Serbia (RTS) distribution main center. In this paper, we have two main contributions. First, we described the influence of existing Long Term Evolution (LTE) technology on wireless microphones which is used in Television stations. Second, it is described how this influence can be reduced using LTE filters searching for optimal filter response.

Keywords:

wireless microphones, LTE Technology, LTE filters, IEEE802.11.
\end{abstract}

\section{INTRODUCTION}

Mobile phones like other wireless devices radiate strong electromagnetic fields that might cause interference and noise in microphone signals. Efforts to correct interference problems are often complicated by the fact that a problem that has been solved usually returns later.

In a technique for improvement of the usage of wireless spectrum in the context of wireless local area networks (WLANs) by using new channel assignment methods among interfering Access Points (APs) was described. They tried to re-use the channel in the realistic interference scenarios in WLAN environments. Two efficient algorithms were proposed that achieved significantly better performance than the state-of-the-art methods. The obtained result was $45.5 \%$ and $56 \%$ reduction in interference for sparse and dense topologies respectively with 3 non-overlapping channels. Additionally, they showed that this approach effectively used partially overlapping channels in order to achieve an additional $42 \%$ reduction on average for moderately sized networks.

A straightforward extension to this work was to handle co-existing $802.11 \mathrm{~b} / \mathrm{g}$ APs in the same area of coverage. However, interference affected 802.11g APs more than 802.11b standard. In [1], the first location oblivious distributed unit disk graph coloring algorithm was proposed. 
This was an improvement over the standard sequential coloring algorithm since they presented a new lower bound of $10 / 3$ for the worst-case performance ratio of the sequential coloring algorithm with the greatest bound of 5/2. However, simulation results showed that this algorithm didn't provide a significant improvement over the previous algorithm which sequentially colored the nodes in an arbitrary order.

In this work, in Section I, we introduce SKM 300-865 G3 wireless microphone that we used in RTS. Section II describes LTE cellular technology that we consider for the purpose of our measurements [2]. In Section III, we analyze the consequences and the problems of interference of LTE systems and wireless microphones working in the same frequency band from $750 \mathrm{MHz}$ to $850 \mathrm{MHz}$. In Section III we presented the measurement results and the results of measurements are concluded giving the issue of implementation and the solution of potential problem.

\section{SKM 300-865 G3 WIRELESS MICROPHONE}

SKM 300-865 G3 presents a super-cardioid handheld microphone/transmitter with condenser microphone capsule and excellent sound quality. Microphone SMK has enhanced frequency bank system up to 24 compatible frequencies and $42 \mathrm{MHz}$ bandwidth. There are 1680 tunable UHF frequencies for interference-free reception, but part of frequency range is between $680 \mathrm{MHz}$ to $830 \mathrm{MHz}$. In order to get all the functionalities that we needed, this wireless microphone was used together with microphone receiver EM3732 and microphone transmitter SK 3036. The EM 3732 is characterized by high transmission reliability, exceptional audio quality and simple operation, switching bandwidth of up to 184 $\mathrm{MHz}$, more than double that of its predecessors. The integrated Ethernet connection means that the receivers can be linked into any network. The AES3/EBU digital audio interface facilitates a direct connection to digital mixing consoles. Sennheiser's "Wireless Systems Manager" (WSM) software allows monitoring and control all receiver operating statuses remotely. In addition, this versatile software enables the channel allocation reset at any time and stores it as a "Scene". The EM 3732 is available in 3 different frequency variants, such as: L: 470 - $638 \mathrm{MHz}, \mathrm{N}: 614$ - $798 \mathrm{MHz}$, and P: 776 - $960 \mathrm{MHz}$.

The SK 3063 is a miniature, tunable bodypack transmitter that provides $30 \mathrm{~mW}$ output power, and $16 \mathrm{se}-$ lectable frequencies in a $24 \mathrm{MHz}$ micro range that operates within the UHF band of 450-960 MHz. The micro range can easily be retuned over a large portion (macro range) of the UHF band to ensure continuous operation regardless of DTV frequency allocations. The HiDyn plus noise reduction system, optimizing attack and decay time, exhibits a signal-to-noise ratio of $108 \mathrm{~dB}(\mathrm{~A})$. Frequency stability is ensured by the use of Phase Lock Loop (PLL) technology. Tunability, ruggedness, and reliability, along with Sennheiser's unsurpassed audio quality. A microphone's directionality or polar pattern indicates how sensitive it is to sounds arriving at different angles about its central axis. The polar patterns represent the locus of points that produce the same signal level output in the microphone if a given sound pressure level (SPL) is generated from that point. How the physical body of the microphone is oriented relative to the diagrams, depends on the microphone design. For large-membrane microphones such as in the Octave, the upward direction in the polar diagram is usually perpendicular to the microphone body, commonly known as "side fire" or "side address". For small size diaphragm microphones such as the Shure, it usually extends from the axis of the microphone commonly known as "end fire" or "top/end address". Super-cardioid microphone design combines several principles in creating the desired polar pattern as it is described in [3].

On the figure 1 super-cardioid wireless microphone is presented.

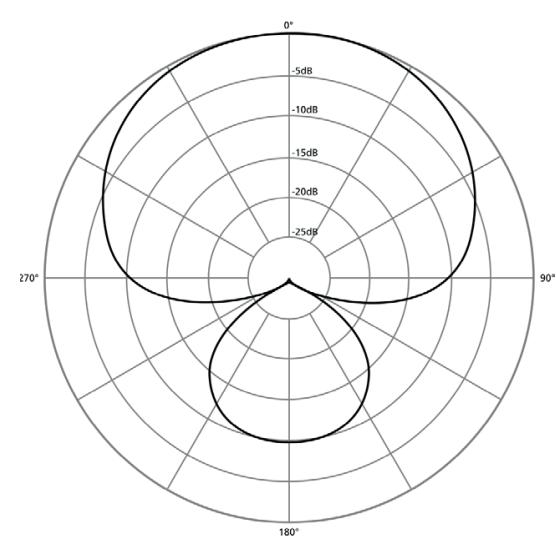

Figure 1. Super-cardioid wireless microphone

\section{LTE TECHNOLOGY}

\section{LTE Technology}

LTE represents a standard for high-speed wireless communication for mobile devices and data terminals, 
based on the GSM/EDGE (Global System for Mobile Communications/ Enhanced Data Rates for GSM Evolution) and UMTS/HSPA (Universal Mobile Telecommunication System/ High Speed Packet Access) technology. The main objectives for LTE are: increased downlink and uplink peak data rates, scalable bandwidth, improved spectral efficiency, all IP network and A standard's based interface that can support a multitude of user types [4]. Serbian base stations are based on Huawei technology. Huawei's 3900 series multi-mode base stations offer a future oriented network solution integrating radio resources and multiple technologies. The design of the 3900 series multi-mode base stations is based on originality that encompasses the latest chip design, system architecture, Power Amplifier (PA) technology, and power consumption management. HUAWEI BTS 3900 is modular cabinet that consist of: RF (Radio Frequency) units (GSM modules, UMTS modules and LTE modules), BBU3900 - unit for digital signal processing, associated cables, DC power distribution unit, fans and ventilation system.

The main point is BBU3900, that can serve to any Radio Access Technologies RATs among GSM, UMTS, and LTE at the same time, thus enabling triple-mode application. Triple-mode is performed by control subsystem using different circuit boards modules [5].

In this work, we use LTE technology, that is able to utilize both Frequency Division Duplex (FDD) and Time Division Duplex (TDD) to accommodate the uplink and downlink. It is essential that any cellular communications system must be able to transmit in both directions simultaneously. Additionally, when exchanging data, it is necessary to be able to undertake virtually simultaneous or completely simultaneous communications in both directions [6].

It is necessary to be able to specify the different direction of transmission, so that it is possible to easily identify in which direction the transmission is made. There is a variety of differences between the two links ranging from the amount of data carried to the transmission format, and the channels implemented. In order to be able to achieve the transmission in both directions, a user equipment or base station must have a duplex scheme.

The frame and subframe structure used within LTE provides the data synchronization and organization required to enable it to be transferred in a logical and ordered fashion. There are several forms of data that need to be sent over the LTE radio interface. LTE uses a series of data channels to provide effective management of the data: physical, logical and transport channels are used.
Those LTE channels provide different interfaces into the higher layers of the protocol stack and in this way they are able to provide efficient management of the data. The physical, logical and transport channels all link to different areas of the stack. By organizing them in this way, the LTE system is able to route the data to the required area. This control channel number so it can be defined easily and its limits known. The LTE radio channels are also allocated numbers - these can be calculated from a simple defined formula. By having defined radio channels, they can be coordinated globally to facilitate roaming [7]. There is a large number of allocations or radio spectrum that has been reserved for FDD, frequency division duplex, and LTE use. The FDD LTE frequency bands are paired to allow simultaneous transmission on two frequencies. Part of FDD LTE Bands and Frequencies are presented In Table 1 . The bands also have a sufficient separation to enable the transmitted signals not to unduly impair the receiver performance. If the signals are too close, then the receiver may be "blocked" and the sensitivity impaired. The separation must be sufficient to enable the roll-off of the antenna filtering to give sufficient attenuation of the transmitted signal within the receive band.

Table 1. Part of FDD LTE Bands \& Frequencies

\begin{tabular}{cccc}
\hline \multirow{2}{*}{$\begin{array}{c}\text { LTE } \\
\text { Number }\end{array}$} & \multicolumn{3}{c}{ Frequency (MHz) } \\
\cline { 2 - 4 } & Uplink & Downlink & $\begin{array}{c}\text { Width } \\
\text { of Band }\end{array}$ \\
\hline 1 & $1920-1980$ & $2110-2170$ & 60 \\
\hline 2 & $1850-1910$ & $1930-1990$ & 60 \\
\hline 3 & $1710-1785$ & $1805-1880$ & 75 \\
\hline 4 & $1710-1785$ & $2110-2155$ & 45 \\
\hline 5 & $824-849$ & $869-894$ & 25 \\
\hline 6 & $830-840$ & $875-885$ & 10 \\
\hline 7 & $2550-2570$ & $2620-2690$ & 70 \\
\hline 8 & $880-915$ & $925-960$ & 35 \\
\hline 9 & $1749.9-1748.9$ & $1844.9-1879.9$ & 35 \\
\hline 10 & $1710-1770$ & $2110-2170$ & 60 \\
\hline 11 & $1427.9-1452.9$ & $1475.9-1500.9$ & 20 \\
\hline 12 & $698-716$ & $728-745$ & 18 \\
\hline 13 & $777-787$ & $745-756$ & 10 \\
\hline 14 & $788-798$ & $758-768$ & 10 \\
\hline 15 & $1900-1920$ & $2600-2620$ & 20 \\
\hline
\end{tabular}




\begin{tabular}{|c|c|c|c|}
\hline \multirow{2}{*}{$\begin{array}{c}\text { LTE } \\
\text { Band } \\
\text { Number }\end{array}$} & \multicolumn{3}{|c|}{ Frequency (MHz) } \\
\hline & Uplink & Downlink & $\begin{array}{l}\text { Width } \\
\text { of Band }\end{array}$ \\
\hline 16 & $2010-2025$ & $2585-2600$ & 15 \\
\hline 17 & $704-716$ & $734-746$ & 12 \\
\hline 18 & $815-830$ & $860-875$ & 15 \\
\hline 19 & $830-845$ & $875-890$ & 15 \\
\hline 20 & $832-862$ & $791-821$ & 30 \\
\hline 21 & $1447.9-1462.9$ & $1495.5-1510.9$ & 15 \\
\hline 22 & $3410-3500$ & $3510-3600$ & 90 \\
\hline 23 & $2000-2020$ & $2180-2200$ & 20 \\
\hline 24 & $1625.5-1660.5$ & $1525-1559$ & 34 \\
\hline 25 & $1850-1915$ & $1930-1955$ & 65 \\
\hline 26 & $814-849$ & $859-894$ & $30 / 40$ \\
\hline 27 & $807-824$ & $852-894$ & 17 \\
\hline 28 & $703-748$ & $758-803$ & 45 \\
\hline 29 & $\mathrm{n} / \mathrm{a}$ & $717-728$ & 11 \\
\hline 30 & $2305-2315$ & $2350-2360$ & 10 \\
\hline 31 & $452.5-457.5$ & $462.5-467.5$ & 5 \\
\hline 32 & DL CA Only & $1452-1496$ & 44 \\
\hline 65 & $1920-2100$ & $2110-2200$ & 90 \\
\hline 66 & $1710-1780$ & $2110-2200$ & 90 \\
\hline 67 & DL CA Only & $738-758$ & 20 \\
\hline
\end{tabular}

\begin{tabular}{ccc}
\hline \multirow{2}{*}{$\begin{array}{c}\text { LTE Band } \\
\text { Number }\end{array}$} & \multicolumn{2}{c}{ Frequency $(\mathrm{MHz})$} \\
\cline { 2 - 3 } & Allocation & Width of Band \\
\hline 39 & $1880-1920$ & 40 \\
\hline 40 & $2300-2400$ & 100 \\
\hline 41 & $2496-2690$ & 194 \\
\hline 42 & $3400-3600$ & 200 \\
\hline 43 & $3600-3800$ & 200 \\
\hline 44 & $703-803$ & 100 \\
\hline 45 & $1447-1467$ & 20 \\
\hline 46 & $5150-5925$ & 775 \\
\hline 47 & $5855-5925$ & 70 \\
\hline
\end{tabular}

There are regular additions to the LTE frequency bands / LTE spectrum allocations as a result of negotiations at the ITU regulatory meetings. These LTE allocations are resulting in part from the digital dividend, and also from the pressure caused by the ever growing need for mobile communications. Many of the new LTE spectrum allocations are relatively small, often 10 $-20 \mathrm{MHz}$ in bandwidth, and this is a cause for concern. With LTE-Advanced needing bandwidths of $100 \mathrm{MHz}$, channel aggregation over a wide set of frequencies many be needed. LTE supports different channel bandwidths and as a result a different number of resource blocks can be supported. For all the channel bandwidths except $1.4 \mathrm{MHz}$, the resource blocks in the transmission bandwidth fill up $90 \%$ of the channel bandwidth.

There are several unpaired frequency allocations that are being prepared for LTE TDD use. The TDD LTE bands are unpaired because the uplink and downlink share the same frequency, being time multiplexed. In Table 2. TDD LTE Bands and Frequencies are presented.

Table 2. TDD LTE Bands \& Frequencies

\begin{tabular}{ccc}
\hline \multirow{2}{*}{$\begin{array}{c}\text { LTE Band } \\
\text { Number }\end{array}$} & \multicolumn{2}{c}{ Frequency $(\mathrm{MHz})$} \\
\cline { 2 - 3 } & Allocation & Width of Band \\
\hline 33 & $1900-1920$ & 20 \\
\hline 34 & $2010-2025$ & 15 \\
\hline 35 & $1850-1910$ & 60 \\
\hline 36 & $1930-1990$ & 60 \\
\hline 37 & $1910-1930$ & 20 \\
\hline 38 & $2570-2620$ & 50 \\
\hline
\end{tabular}

\section{LTE FILTER AND IMPLEMENTATION}

When recording TV programs in RTS studios, wireless microphone signals and sound interruptions were approximate duration from $1 \mathrm{~s}$ to $5 \mathrm{~s}$ over an undetermined time interval. In the Figure 2. the transmission and the reflection parameters are presented.

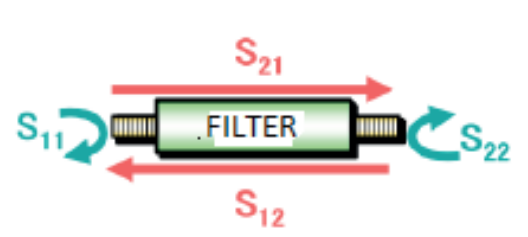

Figure 2. S-parameters 
S-parameters are complex numbers that show Reflection/Tranmission characteristics (Amplitude/Phase) in frequency domain. Our LTE filter is two-port device with four S-parameters (). The numbering convention for S-parameters is that the first number following the " $\mathrm{S}$ " is the port where the signal emerges, and the second number is the port where the signal is applied [8].

With amplitude and phase information (shown in Figure 3), we can quantify the reflection and transmission characteristics of the devices. Some of the common measured terms are scalar (when the phase part is ignored or not measured), while others are vector (when both magnitude and phase are measured). Return loss is a scalar measurement of reflection, while impedance results from a vector reflection measurement. On the other hand, the group delays are purely phase-related measurements [9].

All parameters such as Reflections (Return loss), Impedance, Admittance, and Voltage Vawe Standing Wave Ratio (VSWR). are shown in Figure 3.

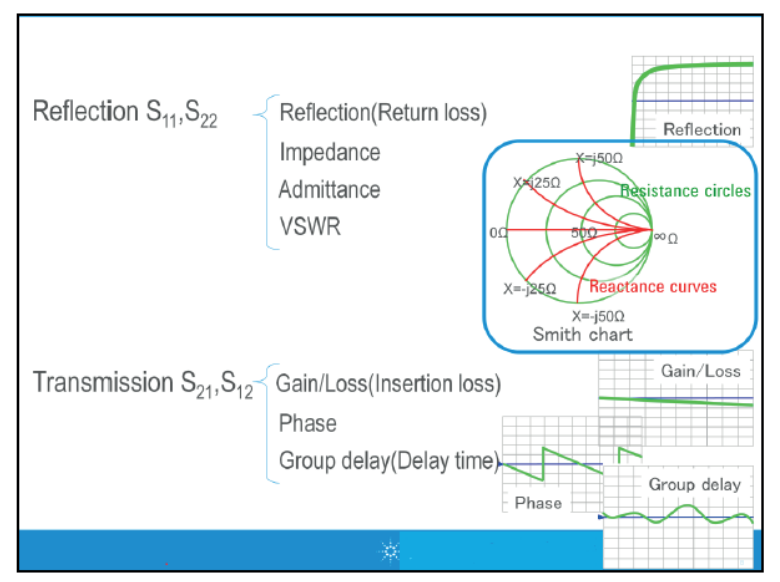

Figure 3. Reflection and transmission parameters

We analyzed the frequency spectrum with Kathrein's MSK 200 signal meter. An LTE filter is designed to stop interference by aggressively decreasing useful signals outside the broadcast band. LTE transmissions occur at several frequencies. In figure 4 and 5, the measurements result with and without implementation of LTE filter in studio Kosutnjak are presented.

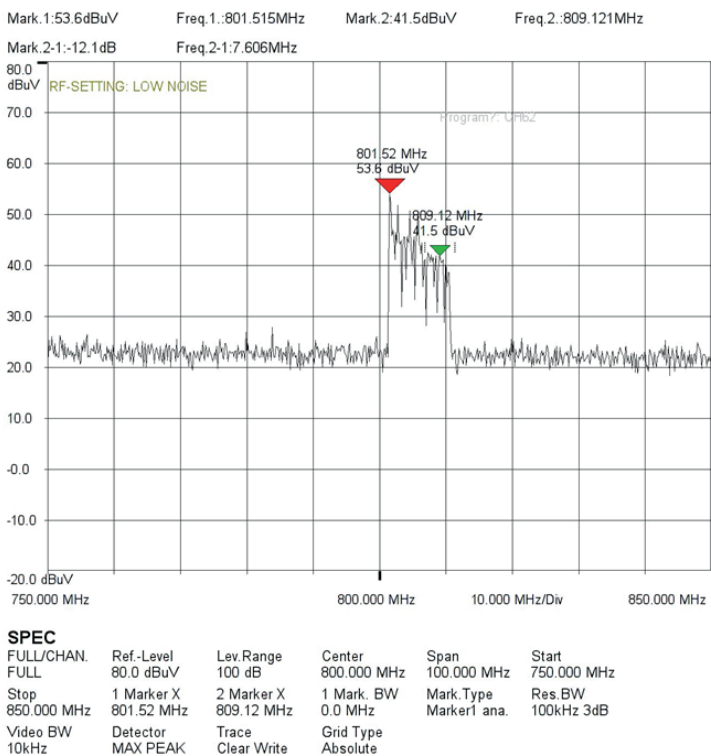

Figure 4. Bandwidth from $750 \mathrm{MHz}$ to $850 \mathrm{MHz}$ in site Kosutnjak

In figure 4 it is shown that LTE signal, in our case the interference signal is up to $40 \mathrm{~dB}$ when wireless microphone up to $790 \mathrm{MHz}$ is used. As it is shown in figure 5, LTE interference signal is very strong and completely masks our wireless microphone signal. As a result, the useful signal on the output from the receiver is missing that resultins in silence.

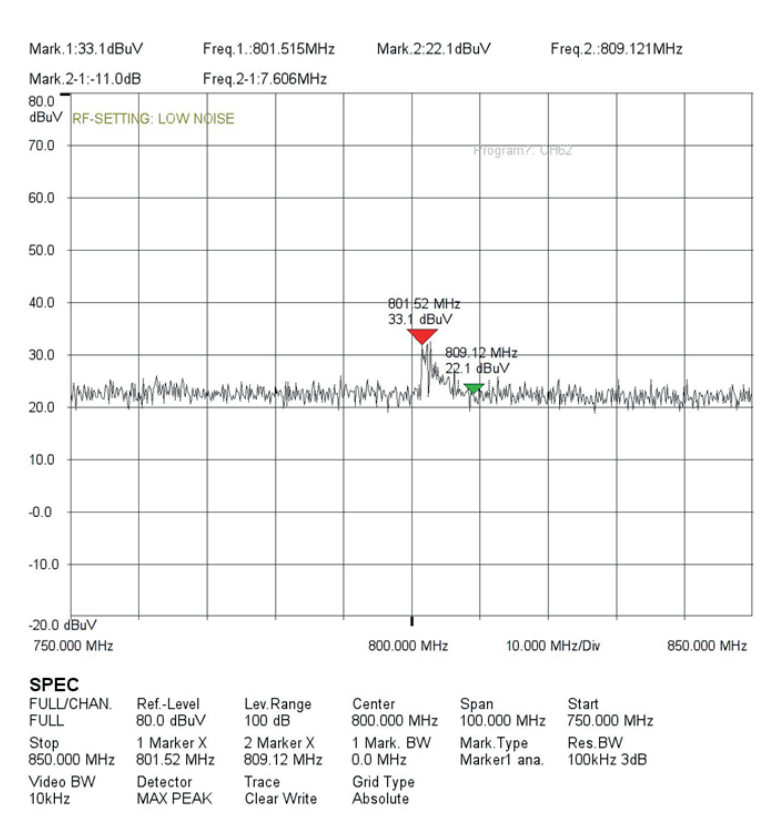

Figure 5. LTE filter implementation in site Kosutnjak 
As it is shown on the Figure 5, we eliminate the LTE unused signal, but in bandwidths from $400 \mathrm{MHz}$ to $820 \mathrm{MHz}$ S12 parameter is too high, up to $20 \mathrm{db}$. That means that our wireless microphone signal will be attenuated up to $20 \mathrm{~dB}$. Thus, we create the adapted filter (SLTE30) to the needs of a recording studio with a very small attenuation of the parameter S12 that is not to degrading the useful signal as it is shown on Figure 6. where S21 parameter is up to $3 \mathrm{db}$.

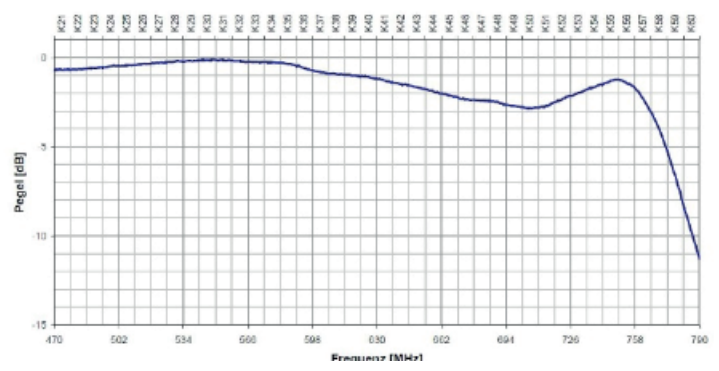

Figure 6. Insertion loss vs. frequency for SLTE30 filter

Therefore, microphone wireless signal in microphone receiver without LTE signal and attenuation microphone signal is very low and acceptable.

\section{CONCLUSION}

In this paper, the measured LTE interference between wireless microphone signal and the LTE $4 \mathrm{G}$ signal from mobile operator is presented. It is shown that interference wireless signal and LTE signal when recording a TV live show brings only silence. There is no music, and no votes. In cooperation with mobile operators, we determine the source of LTE signal. Additionally, we analyze the bandwidth $(750-850 \mathrm{MHz}$ where our wireless microphone work and interference LTE signal occurs. Finally, we solve this problem and eliminate interference of LTE signal. In a first phase, we use the commercial LTE filters and we eliminate the interference. However, using the commercial LTE filters we also damage the useful wireless microphone signal. Thus, after the analysis of used filters, various adjustments, experiments modifications and we created a new LTE filter that eliminates the interference of LTE signal and transmits signal without any distortion.

\section{REFERENCES}

[1] IEEE 802.11tm wireless local area networks, Available at: http://www.ieee802.org/11/, access to 25.02.2019

[2] J. Beneasty,C. Jingdong, Study and Design of Differential Microphone Arrays, Springer Sciences Business Media., 9783642337429, 2012.

[3] L. Zhao, J. Benesty,C. Jihdong, Design of robust differential microphone arrays with the Jacobi-Anger expansion, Available at: https://doi.org/10.1016/j, , access to 05.03.2019

[4] Huawei technologies co., ltd 3900 Series Base stetion, September 2011

[5] IEEE 802.11-2016 - IEEE Standard for Information technology,Telecommunications and information exchange between systems Local and metropolitan area networks--Specific requirements - Part 11: Wireless LAN Medium Access Control (MAC) and Physical Layer (PHY) Specifications, Available at: https://standards.ieee.org/standard/802_11-2016. html, access to 07.03.2019

[6] C. Cox, An Introduction to LTE: LTE, LTE-Advanced, SAE, VoLTE and 4G Mobile Communications , July 2014.

[7] An Introduction to LTE, 3GPP LTE Encyclopedia, December 2010.

[8] P. J. Pupalaikis, Validation methods for S-parameter measurement based models of differential transmission lines, Santa Clara, Februar 2008.

[9] A. B. Williams, Analog Filter and Circuit Design Handbook, McGraw-Hill Education, 97800 71816717, 2014 